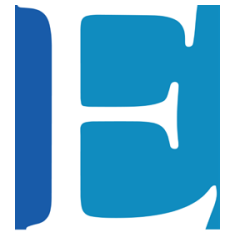

EVALUAR
2018, Vol. 18, No 1.

ISSN 1667-4545

Recuperado de https://revistas.unc.edu.ar/index.php/revaluar

Laboratorio de Evaluación Psicológica y Educativa

Facultad de Psicología - Universidad Nacional de Córdoba

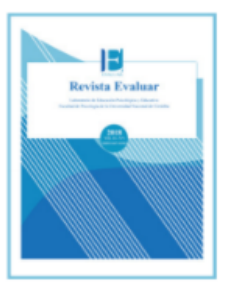

\title{
Validación de una Escala sobre Actitudes hacia la Historia para estudiantes de Secundaria
}

\author{
Validation of a Scale for measuring Attitudes towards History \\ in Secondary students
}

${\text { Lara Yáñez-Marquina * }{ }^{1} \text {,Visitación Pereda }{ }^{1}, \text { Ricardo Palmeiro }^{2}, \text { Erlantz Velasco }{ }^{1}}^{\begin{array}{l}\text { Método } \\ \text { Resultados } \\ \text { Discusión } \\ \text { Referencias }\end{array}}$

1 - Universidad de Deusto, Bilbao.

2 - Bilbao (España).

Recibido: 26/09/2017 Revisado: 7/12/2017 Aceptado: 4/01/2018

\section{Resumen}

Diversas investigaciones constatan la importancia del efecto mediador de las actitudes en la construcción del saber histórico. Sin embargo, pese a dicha constatación, la revisión de la literatura indica la existencia de un único instrumento para la medición del constructo actitudes hacia la historia, validado con técnicas exploratorias en contexto anglosajón. La presente investigación tiene por objetivo diseñar y validar, mediante técnicas confirmatorias, una escala más completa para su empleo en un contexto de habla hispana. La muestra estuvo constituida por 430 estudiantes, con una edad media de $16.29(\mathrm{DE}=1.02)$ años. Los resultados confirmaron que la estructura, de 14 ítems, arrojó un buen ajuste de modelo $\left(\mathrm{S}-\mathrm{B} \chi^{2}(74, \mathrm{~N}=430)=215.54, \mathrm{p}<\right.$ .001 ; RMSEA [90\% IC] $=.068[.058-.079]$; NNFI $=.94$; $\mathrm{CFI}=.95)$. La variable actitudes hacia la historia resulta un constructo de dos componentes, uno de primer orden y otro de segundo, que comprenden un total de tres factores: utilidad e importancia percibida, interés hacia el aprendizaje histórico y creencias conservadoras.

Palabras clave: actitudes hacia la historia, análisis factorial confirmatorio, escala, enseñanza secundaria

\begin{abstract}
Several studies confirm the relevance of the mediating effect of attitudes in the construction of historical knowledge. However, despite this evidence, a literature review indicates the existence of a single instrument for measuring attitudes toward History, validated through exploratory approaches in English-speaking contexts. Therefore, the aim of this paper is to design and validate, through confirmatory techniques, a more completed scale for its use in Spanish-speaking contexts. Data were collected from 430 students, with an average age of 16.29 $(\mathrm{SD}=1.02)$ years. The results confirmed that the 14-item structure showed a good fit to the data $(\mathrm{S}-\mathrm{B} \chi 2(74, \mathrm{~N}=430)$ $=215.54, \mathrm{p}<.001$; RMSEA $[90 \% \mathrm{CI}]=.068$ [.058-.079]; $\mathrm{NNFI}=.94$; CFI $=.95)$. Attitudes toward History is a twocomponent construct, comprising three underlying factors: perceived usefulness and utility, interest for historical knowledge and conservative beliefs.
\end{abstract}

Keywords: attitudes toward History, confirmatory factor analysis, scale, secondary education 


\section{Introducción}

Las actitudes forman parte fundamental de los procesos de aprendizaje de las diferentes materias y disciplinas escolares. En la actualidad, diferentes investigaciones revelan su efecto mediador en la construcción de los diferentes conocimientos escolares.

No obstante, a diferencia del importante bagaje empírico en el análisis de las actitudes en áreas o espacios disciplinarios como las matemáticas (Pietsch, Walker, \& Chapman, 2003; Tarraga, 2008; Zan, Brown, Evans, \& Hannula 2006), la música (Bautista \& Mateos, 2011; Teitsma, 2010) o las lenguas (Huguet, 2005; Lasagabaster, 2003), la revisión de la literatura revela un importante vacío en el espacio de investigación del área de la historia (Burke, 1984) a pesar de la indudable importancia del conocimiento histórico, tanto en el ámbito escolar como en muchos otros de la vida, y de la constatación de que existe una disposición negativa entre los estudiantes hacia esta asignatura (Casal, 2011; Fuentes-Moreno, 2002).

La aproximación desde perspectivas empíricas a este campo se ha limitado fundamentalmente al ámbito anglosajón (Barton, 2010; Burke, 1984; Von Borries, 1994) y a comunidades en las que históricamente ha existido algún tipo de conflicto relacionado con la identidad nacional (Kölbl \& Straub, 2001), conflictos raciales (Wertsch, 1994) e incluso conflictos lingüísticos (Létorneau \& Moisan, 2004). En el ámbito de España no se han encontrado trabajos de este tipo ni escalas que propongan una validación en castellano.

En efecto, una revisión de la literatura ha constatado la existencia de un único instrumento para la medición de actitudes hacia la historia. Se trata de la escala desarrollada y validada por
Andrews, McGlynn y Mycock (2009, 2010). En este trabajo, sus autores únicamente consideran la inclusión del componente cognitivo del constructo actitudes a través de los factores que denominan conservador o tradicional y liberal o multicultural.

Aunque el instrumento desarrollado y validado por estos autores responde a un modelo unidimensional, constituido por factores de corte meramente cognitivo, la revisión de la literatura evidencia la existencia de tres modelos para la conceptualización teórica del constructo actitudes: un modelo unidimensional (Fishbein, 1963, 1967; Thurstone, 1928), un modelo tripartito (Rosenberg \& Hovland, 1960) y un modelo de dos componentes (Bagozzi \& Burnkrant, 1979).

Por una parte, el modelo unidimensional resulta ser la primera propuesta estructural para la conceptualización de las actitudes existente en la literatura, que fue desarrollada originalmente por Thurstone (1928) y retomada posteriormente por Fishbein (1936, 1967). De acuerdo a este marco, el constructo únicamente considera la inclusión de afectos positivos o negativos como producto de las creencias y la evaluación personal que un individuo realiza del objeto actitudinal, que en este caso es la historia. Sin embargo, diversos estudios posteriores mostraron la insuficiencia de esta conceptualización para explicar con éxito las actitudes del sujeto (Bettman, Capon, \& Lutz, 1975; Hawkins, Mothersbaugh, \& Best, 2007; Mazis, Ahtola, \& Klippel, 1975; Sheth, 1974; Tuncalp \& Sheth, 1975; Wilkie \& Pessemier, 1973).

Como respuesta a las limitaciones del modelo unidimensional, y dada la relevancia de las creencias en el proceso de génesis y desarrollo de actitudes, así como la manifestación de las mismas a través de las actuaciones del individuo, Rosenberg y Hovland (1960) sugieren la inclusión de dos nuevos componentes. Estos autores 
sugieren incluir el componente cognitivo y el conativo como parte constituyente del constructo, junto con el ya considerado componente afectivo, dando lugar así al denominado modelo tripartito. Dicho marco teórico ha gozado de gran aceptación en la comunidad científica (Breckler, 1984; Kothandapani, 1971; Ostrom, 1969; Woodsmansee \& Cook, 1967) pero el desarrollo reciente de las técnicas confirmatorias en el ámbito de las ciencias sociales ha demostrado dos debilidades del modelo en aquellos estudios en los que las actitudes han sido conceptualizadas como un constructo tridimensional. En primer lugar, el análisis de su estructura interna ha demostrado altas correlaciones entre los tres factores propuestos, lo que dificulta asegurar su validez discriminante (Bagozzi, Tybout, Craig, \& Sternthal, 1979; McGuire, 1989). En segundo lugar, diversos estudios enfocados en explicar el cambio actitudinal y la predicción comportamental han mostrado una débil relación entre actitud y comportamiento (Ajzen, 1989; Fishbein \& Ajzen, 1975; McGuire, 1989).

Por estos motivos, las debilidades del modelo unidimensional para la conceptualización del constructo actitudes (p. ej., Hawkins, et al. 2007) y la débil relación de causalidad entre componente conativo y actitud (p. ej., McGuire, 1989), Bagozzi y Burnkrant (1979) sugieren la adopción de un modelo de dos componentes, uno afectivo y otro cognitivo. De acuerdo a este modelo, la acción conjunta de ambos componentes lleva al individuo a adoptar una serie de intenciones comportamentales, que definen posteriormente su comportamiento respecto al objeto actitudinal.

De este modo, teniendo en cuenta no sólo el planteamiento teórico de los posibles modelos para las actitudes sino también los resultados empíricos, tanto de naturaleza correlacional como confirmatoria, se ha elegido el marco de dos componentes (Bagozzi \& Burnkrant, 1979) como la conceptualización teórica de base para definir el constructo actitudes hacia la historia en el presente trabajo. De acuerdo a este marco referencial, tanto las dimensiones afectivas como cognitivas llevan al individuo a adoptar una serie de intenciones comportamentales que definen posteriormente su comportamiento hacia el objeto actitudinal. La investigación empírica llevada a cabo tras el planteamiento de este modelo teórico confirma ampliamente una adecuada validez convergente, discriminante y predictiva de la estructura factorial propuesta para el constructo (Esses, Haddock, \& Zanna, 1993; Huskinson \& Haddock, 2006; Kraus, 1995; Millar \& Millar, 1990; Verplanken, Hofstee, \& Janssen, 1998).

Como se ha referido anteriormente, si bien la escala de Andrews et al. (2009, 2010) se considera un gran aporte al estudio de las actitudes hacia la historia, cabe destacar que los estudios de validación realizados hasta el momento únicamente abarcan un contexto anglosajón, y no se han encontrado evidencias de su robustez psicométrica en contextos de habla hispana. Asimismo, sus autores únicamente han hecho uso de técnicas exploratorias para analizar su confiabilidad y consistencia interna. Es por ello que la presente investigación tiene por objetivo desarrollar y validar, mediante técnicas confirmatorias, en un contexto de habla hispana, un nuevo instrumento para la medición de actitudes hacia la historia que responda al modelo de dos componentes.

Así, tomando como base este marco conceptual, el modelo teórico propuesto abarca tres factores latentes de primer orden que se reagrupan para dar lugar a un modelo de dos componentes, uno de primer orden (referido al componente afectivo, explicado a partir de la motivación hacia el aprendizaje histórico) y otro de segundo orden (referido al componente cognitivo, que abarca a los factores de primer 
orden utilidad e importancia percibida y creencias conservadoras):

Utilidad e importancia percibida corresponde a la dimensión cognitiva y mide el valor de utilidad extrínseca que el individuo otorga a la historia, es decir, las creencias de aplicabilidad del saber histórico en su vida personal y futura carrera profesional (p. ej., No creo que los temas que estudiamos en Historia sean importantes para mi futuro).

Creencias conservadoras corresponde a la dimensión cognitiva y mide el grado de acuerdo del individuo hacia actuaciones conservadoras ( $\mathrm{p}$. ej., El Estado debería tener el control sobre lo que se enseña en las clases de Historia para asegurar que todos los ciudadanos tendrán la misma visión de la identidad nacional y el mismo sentido de lealtad hacia el país).

Motivación hacia el aprendizaje histórico corresponde a la dimensión afectiva y mide el nivel de motivación del individuo a la hora de aprender y compartir conocimientos históricos ( $\mathrm{p}$. ej., Disfruto debatiendo sobre temas históricos).

\section{Método}

\section{Participantes}

La muestra estuvo constituida por 430 estudiantes procedentes de la Comunidad Autónoma de País Vasco que cursaban la asignatura de Historia en $4^{\circ}$ curso de Educación Secundaria Obligatoria (E.S.O.) y $2^{\circ}$ curso de Bachillerato seleccionados mediante un muestreo por conglomerados. La edad media fue de 16.29 $(\mathrm{DE}=1.02)$ años, con un rango que oscilaba entre 14 y 19 años. El porcentaje de mujeres fue 45.1\%; mientras que el de hombres fue 54.8\%. La Tabla 1 muestra la distribución de los participantes.
Tabla 1

Distribución de la muestra.

\begin{tabular}{llll}
\hline & Mujeres & Hombres & Total \\
\hline $4^{\circ}$ E.S.O. & 79 & 69 & 148 \\
$2^{\circ}$ Bachillerato & 115 & 167 & 282 \\
Total & 194 & 236 & 430 \\
\hline
\end{tabular}

\section{Instrumentos}

Escala sobre actitudes hacia la historia. Teniendo en cuenta el marco conceptual del modelo de dos componentes, según el cual actitudes engloba dos dimensiones, que son cognitiva y afectiva, la escala validada en la presente investigación para un contexto de habla hispana se diseñó a partir de dos instrumentos existentes en la literatura: Actitudes hacia la Historia (Andrews et al., 2009, 2010), que engloba únicamente factores de naturaleza cognitiva; y Escala de Motivación Académica (Aydin, Yerdelen, Gürbüzoğlu-Yalmanci, \& Göksu, 2014), que engloba únicamente factores de naturaleza afectiva. La elección de este segundo instrumento para complementar la estructura de Actitudes hacia la Historia se justifica en las evidencias recopiladas en estudios anteriores, según las cuales la motivación, dimensión de carácter afectivo, resulta un factor constituyente del constructo actitudes (p. ej., Auzmendi, 1992; Fennema \& Sherman, 1976; Gairín, 1990; Lim \& Chapman, 2013; Sandman, 1980; Tapia \& Marsch, 2004).

Por una parte, el instrumento de Andrews et al. $(2009,2010)$ consta de 7 ítems distribuidos en dos factores independientes, que son tradicional/conservador y multicultural/ liberal. Los participantes puntúan su grado de acuerdo con cada ítem en una escala Likert de 
5 puntos, desde Completamente en Desacuerdo (1) a Completamente de Acuerdo (5). Los ítems propuestos por Andrews et al. (2009) muestran cargas factoriales superiores a .52 y el porcentaje de varianza total acumulada es $50.4 \%$.

Por su parte, el instrumento de Aydin et al. (2014) es una escala con 19 ítems distribuidos en cuatro factores independientes, que incluyen amotivación, motivación extrínseca relativa a la carrera profesional, motivación extrínseca relativa a la proyección social y motivación intrínseca. En este caso, los participantes puntúan su grado de acuerdo con cada ítem en una escala Likert de 6 puntos, desde Completamente en Desacuerdo (1) a Completamente de Acuerdo (6). En el estudio original de Aydin et al. (2014) se han verificado para el instrumento buenos coeficientes de consistencia interna, con valores alfa de Cronbach comprendidos entre $\alpha=.73$ y $\alpha$ $=.88$ para cada una de las subescalas. Asimismo, cabe destacar que los ítems están redactados para medir los niveles de motivación en las 4 subescalas en relación con el aprendizaje de Biología, por lo que en la presente investigación, durante el proceso de traducción y adaptación al castellano, se sustituyeron en la redacción de los ítems las referencias a Biología por Historia.

Tomando como base dichos instrumentos, se eliminaron los ítems redundantes y se redistribuyeron los restantes de acuerdo a las categorías teóricamente hipotetizadas para la nueva escala: utilidad e importancia percibida (5 ítems), motivación hacia el aprendizaje histórico (5 ítems) y creencias conservadoras ( 7 ítems; véase Apéndice 1). El proceso de validación se realizó ampliando el rango de respuesta a una escala continua desde 0 (Completamente en Desacuerdo) hasta 10 (Completamente de Acuerdo). La elección de tal rango de respuesta para la presente investigación radica en que su empleo permite conocer con mayor precisión el grado de acuerdo del participante para cada ítem, lo que a su vez aporta mayor robustez y confiabilidad a los consecuentes análisis estadísticos (Pett, Lackey, \& Sullivan, 2003).

Cuestionario sociodemográfico. Se administró un cuestionario ad hoc para recopilar información sociodemográfica sobre los estudiantes participantes en el estudio (edad, sexo, curso).

\section{Procedimiento}

Una vez que el Comité Ético de la Universidad de Deusto aprobó el proyecto de investigación, se procedió a la traducción y adaptación de las escalas de origen al contexto objeto de la presente investigación. Para ello, se llevó a cabo un proceso de traducción por comité (Marin \& Marin, 1991) y se siguieron las recomendaciones de la Comisión Internacional para la Evaluación (Muñiz, Elosua, \& Hambleton, 2013), buscando conservar el sentido psicológico de cada ítem y no el literal. Asimismo, se adaptó la terminología empleada para que resultase familiar a la población referencia del presente estudio (Clark \& Watson, 1995; DeVellis, 2012), consistente en estudiantes de Secundaria con edades comprendidas entre los 14 y 19 años. Los traductores trabajaron de manera independiente y se compararon las versiones elaboradas, alcanzando el acuerdo para la totalidad de los ítems que compondrían la versión inicial de la escala.

Posteriormente, se contactó vía e-mail al equipo directivo de los centros educativos seleccionados, los cuales, a su vez, presentaron el instrumento al resto del equipo docente para su aprobación. Tras obtener el permiso de los centros, las familias y/o tutores legales de los estudiantes fueron informados del propósito de la investigación y se les explicó que los datos 
recogidos serían tratados de manera confidencial y con fines exclusivamente científicos, tal y como queda establecido por la Ley Orgánica 15/1999 de Protección de Datos de Carácter Personal. Se les solicitó la autorización firmada y se facilitaron los datos de contacto de los autores para el caso de que tuvieran dudas o precisaran información adicional sobre el estudio.

Finalmente, los estudiantes fueron invitados a participar de manera anónima y voluntaria y se les recordó su derecho a abandonar el estudio en cualquier momento si lo consideraban necesario. Todos los estudiantes de la muestra aportaron $\mathrm{su}$ consentimiento informado tanto para su participación como para el tratamiento de los datos en el marco de la investigación. Así, el instrumento fue administrado por los autores de manera colectiva en las aulas de los estudiantes y en ausencia del profesor.

\section{Análisis de datos}

Con objeto de adaptar consistentemente la escala sobre actitudes hacia la historia al contexto de la comunidad autónoma del País Vasco, se realizaron una serie de análisis estadísticos con los softwares SPSS Versión 22 (IBM, 2013) y EQS 6.1 (Multivariate Software, 2012).

En primer lugar, tras la administración de la escala a la muestra y la posterior recogida de datos, se recodificaron los ítems con código inverso, se analizó la presencia de valores perdidos y outliers, y se comprobaron los supuestos estadísticos para el posterior análisis factorial confirmatorio (en adelante AFC; Tabachnick \& Fidell, 2007). Por una parte, la normalidad univariada se evaluó en términos de asimetría y curtosis, asumiendo que coeficientes superiores a 2.30 en valor absoluto son indicativos de divergencia con respecto a una distribución normal (Lei \& Lomax, 2005). Por otra parte, la normalidad multivariada se analizó a través del coeficiente estandarizado de Mardia, para el que se estableció como criterio un valor máximo de 5 (Bentler, 2005; Ullman, 2006).

Así, tras los análisis preliminares de los ítems y con objeto de investigar la validez de constructo, se efectuó el AFC sobre la estructura factorial hipotetizada. Esta técnica se prefirió sobre un análisis factorial exploratorio (en adelante, AFE) ya que se buscaba probar la estructura propuesta a partir de los estudios de la literatura, los cuales hasta el momento habían sido abordados únicamente con una aproximación exploratoria. La normalidad univariada obtenida para todos los ítems, el bajo porcentaje de valores perdidos $(<$ $5 \%$ ) y el tamaño de la muestra empleada en la presente investigación llevaron a la selección del método de estimación por máxima verosimilitud (Chou \& Bentler, 1995; Finney \& DiStefano, 2006) y eliminación por lista (Brown, 1983, 2006).

Por su parte, la falta de normalidad multivariada sugirió la estimación de los parámetros del AFC mediante correcciones robustas de Satorra-Bentler (S-B $\chi^{2}$; Satorra, 2003; Satorra \& Bentler, 2001). Se forzó la inclusión de los ítems en los factores hipotetizados. Las varianzas para el primer indicador observado de cada variable latente fueron fijadas en 1, y las varianzas para los errores y el resto de parámetros fueron libremente estimadas (U1lman, 2006).

Los análisis se efectuaron en dos fases. La primera consistió en efectuar el AFC para la estructura teórica propuesta; mientras que la segunda fase tuvo por objeto implementar mejoras en la estructura factorial. Se utilizaron una serie de índices de bondad de ajuste que permitieron evaluar la adecuación del modelo (Hair, Black, Babin, \& Anderson, 2010): a) el ratio S-B $\chi^{2} / g l$ debe ser inferior a 3.00 (Bentler, 2005; Bollen, 1989); b) el valor de la raíz del error cuadrático 
medio de aproximación (RMSEA), con un intervalo de confianza de $90 \%$, debe ser inferior a .08 (Browne, MacCallum, Kim, Andersen, \& Glaser, 2002; Hu \& Bentler, 1999); c) el índice de ajuste no normalizado (NNFI) debe ser superior a .90 (Hoyle \& Panter, 1995); y d) el índice de ajuste comparativo (CFI,) debe ser superior a .90 (Bentler, 1990; Kline, 2005; Marsh, Hau, \& Wen, 2004). Asimismo, se consideró el criterio de información de Akaike (AIC,) para evaluar la estructura más parsimoniosa $(\mathrm{Hu} \&$ Bentler, 1999; Kline, 2005).

A continuación se analizó la posibilidad de implementar mejoras en el modelo, para lo que se inspeccionaron los coeficientes de covarianza residuales estandarizados, la matriz de correlaciones inter-ítem y las cargas factoriales estandarizadas. En concreto, se consideró que valores residuales estandarizados inferiores a 2.00 en valor absoluto, correlaciones inter-ítem inferiores a .30 y cargas factoriales por debajo de .40 eran indicativos de bajo comportamiento psicométrico (Floyd \& Widaman, 1995).

La consistencia interna fue evaluada con los coeficientes confiabilidad compuesta (CR) y alfa de Cronbach $(\alpha)$. Por su parte, para evaluar la validez discriminante se comprobó que el CR de cada factor fuese superior a los coeficientes de correlación de dicho factor con el resto (Bagozzi \& Yi, 2012; Henseler, Ringle, \& Sarstedt, 2015). Finalmente, la validez convergente se estableció a través de la varianza media explicada (AVE), estableciendo como valores adecuados aquellos superiores a .50 (Fornell \& Larcker, 1981).

\section{Resultados}

Con respecto a la asunción de normalidad, esta fue examinada en dos fases. En primer lugar, se analizó la normalidad univariada a través de los coeficientes de asimetría y curtosis (Tabla 2). Los estadísticos descriptivos mostraron que todos los ítems cumplían el criterio establecido.

Tabla 2

Valores de asimetría y curtosis para cada ítem de la escala.

\begin{tabular}{|c|c|c|c|c|c|c|c|}
\hline Ítem & $\mathbf{N}$ & Mín. & Máx. & $\mathbf{M}$ & DE & Asimetría & Curtosis \\
\hline IT01 & 418 & 0 & 10 & 6.63 & 2.05 & -0.83 & 1.30 \\
\hline IT02 & 430 & 0 & 10 & 5.81 & 2.69 & -0.50 & -0.56 \\
\hline IT03 & 426 & 0 & 10 & 6.86 & 2.97 & -0.81 & -0.45 \\
\hline IT04 & 428 & 0 & 10 & 3.82 & 2.92 & 0.39 & -0.95 \\
\hline IT05 & 429 & 0 & 10 & 5.56 & 2.71 & -0.39 & -0.60 \\
\hline IT06 & 424 & 0 & 10 & 5.23 & 2.55 & -0.39 & -0.52 \\
\hline IT07 & 426 & 0 & 10 & 6.60 & 3.21 & -0.69 & -0.76 \\
\hline IT08 & 427 & 0 & 10 & 4.42 & 3.22 & 0.05 & -1.21 \\
\hline IT09 & 429 & 0 & 10 & 6.59 & 2.66 & -0.89 & 0.13 \\
\hline IT10 & 430 & 0 & 10 & 5.55 & 2.81 & -0.39 & -0.74 \\
\hline IT11 & 428 & 0 & 10 & 3.99 & 3.06 & 0.12 & -1.19 \\
\hline IT12 & 429 & 0 & 10 & 2.92 & 2.96 & 0.70 & -0.59 \\
\hline IT13 & 429 & 0 & 10 & 2.78 & 2.79 & 0.67 & -0.67 \\
\hline IT14 & 427 & 0 & 10 & 5.93 & 3.15 & -0.31 & -1.13 \\
\hline IT15 & 425 & 0 & 10 & 3.58 & 3.00 & 0.32 & -1.01 \\
\hline IT16 & 428 & 0 & 10 & 6.04 & 2.77 & -0.59 & -0.44 \\
\hline IT17 & 428 & 0 & 10 & 6.39 & 2.78 & -0.52 & -0.64 \\
\hline
\end{tabular}

normalidad multivariada a través del coeficiente estandarizado de Mardia, que resultó ser 21.60 $(p<.001)$, por lo que se optó por implementar métodos robustos para la estimación de errores estandarizados, errores estadísticos e índices de bondad de ajuste (Satorra, 2003; Satorra \& Bentler, 2001).

Con estas consideraciones se sometió el modelo teórico, de tres factores relacionados de primer orden (M1), a AFC. Los resultados sugirieron un buen ajuste del modelo $\left(\mathrm{S}-\mathrm{B} \chi^{2}{ }_{(116,}\right.$ ${ }_{\mathrm{N}=430)}=396.03, p<.001$; RMSEA [90\% IC] $=$ $.078[.070, .087] ; \mathrm{NNFI}=.88 ; \mathrm{CFI}=.90 ; \mathrm{AIC}$ $=164.03)$. En efecto, a excepción del NNFI, que resultó inferior al valor deseable de .90 , el resto de los índices de bondad de ajuste cumplieron con los criterios previamente establecidos. 
Con objeto de analizar la posibilidad de implementar mejoras al modelo, se inspeccionaron las cargas factoriales estandarizadas, los índices de covarianza y los coeficientes de covarianza residual estandarizados. Por una parte, las cargas factoriales (Tabla 3) resultaron estadísticamente significativas a nivel $p<.05$ y superiores a .58 , a excepción de IT01 (Las minorías étnicas deberían ser apoyadas para que preserven su historia y su cultura, y ello debería quedar reflejado en las clases de Historia), IT06 (La inmigración y la globalización han reforzado mi comprensión del lugar donde vivo) e IT08 (Los inmigrantes que buscan la nacionalidad deberian pasar una prueba sobre nuestra historia cultural y nacional). Este resultado, junto con el análisis de la matriz de coeficientes de covarianza residual estandarizados, sugirió la eliminación de dichos ítems. Por su parte, la inspección de los índices de modificación no sugirió forzar la covarianza de ningún ítem.

Tabla 3

Cargas factoriales estandarizadas en el modelo teórico (M1).

\begin{tabular}{llll}
\hline Factor & Ítem & \multicolumn{1}{l}{$\boldsymbol{\lambda}$} & e \\
\hline \multirow{3}{*}{ Utilidad e importancia } & IT03 & .89 & .45 \\
percibida & IT07 & .84 & .54 \\
& IT14 & .64 & .77 \\
& IT17 & .58 & .81 \\
& IT04 & .60 & .80 \\
Motivación hacia el & IT16 & .91 & .41 \\
aprendizaje histórico & IT05 & .90 & .43 \\
& IT10 & .83 & .56 \\
& IT09 & .77 & .64 \\
& IT02 & .80 & .60 \\
Creencias conservadoras & IT12 & .76 & .65 \\
& IT13 & .75 & .67 \\
& IT15 & .73 & .68 \\
& IT11 & .66 & .75 \\
& IT01 & .07 & .99 \\
& IT06 & .20 & .98 \\
& IT08 & .32 & .95 \\
\hline
\end{tabular}

Por lo tanto, se procedió a realizar la mencionada modificación sobre el modelo (MIa) y se recalcularon los índices, comprobando si la diferencia en $\mathrm{S}-\mathrm{B} \chi^{2}$ resultaba significativa con respecto al modelo teórico $(M 1)$. La Tabla 4 muestra que el modelo Mla presentaba un mejor ajuste, con un menor ratio $\mathrm{S}-\mathrm{B} \chi^{2} / g l$, y unos mayores coeficientes para NNFI y CFI, resultados que se consideran excelentes. Asimismo, tanto el valor RMSEA como su intervalo de confianza fueron inferiores a .08, y el AIC sugirió que se trataba de una estructura más parsimoniosa. De este modo, la eliminación de los tres ítems mencionados resultó en una mejora del modelo estadísticamente significativa $\left(\Delta \mathrm{S}-\mathrm{B} \chi^{2}=180.49\right.$, $\Delta g l=42, p<.001)$.

Tabla 4

Índices de bondad de ajuste para el modelo teórico (M1) y modelo modificado (M1a).

\begin{tabular}{|c|c|c|c|c|c|c|}
\hline Modelo & $\begin{array}{l}\mathrm{S}-\mathrm{B} \chi^{2} \\
/ \mathbf{g l}\end{array}$ & $\begin{array}{l}\text { RMSEA } \\
(90 \% \text { IC) }\end{array}$ & NNFI & CFI & AIC & $\begin{array}{l}\Delta \mathrm{S}-\mathrm{B} \chi^{2} \\
(\Delta \mathrm{gl})\end{array}$ \\
\hline M1 & 3.41 & $\begin{array}{l}0.078 \\
{[0.070-} \\
0.087]\end{array}$ & .88 & .90 & 164.03 & / \\
\hline M1a & 2.91 & $\begin{array}{l}0.068 \\
{[0.058-} \\
0.079]\end{array}$ & .94 & .95 & 67.54 & $\begin{array}{l}180.49 * * * \\
(42)\end{array}$ \\
\hline
\end{tabular}

Por último, se analizó la confiabilidad del modelo final (Figura 1). Los resultados arrojaron buenos índices tanto para la escala completa $(\alpha=.77)$ como para cada una de las dimensiones consideradas: utilidad e importancia percibida $(\mathrm{CR}=$ .84), motivación hacia el aprendizaje histórico $(\mathrm{CR}=.93)$ y creencias conservadoras $(\mathrm{CR}=.81)$. Asimismo, como se observa en la Tabla 5, el índice de confiabilidad compuesta de cada factor resultó superior a los valores de las correlaciones entre este y el resto de los factores considerados, lo que aseguró la validez discriminante de la escala. Finalmente, los valores AVE resultaron supe- 
riores a .50 , lo que indicó que los factores incluidos en el modelo lograron explicar más del 50\% de la varianza del constructo.

\section{Figura 1}

Modelo final de la escala $(\mathrm{N}=430)$.

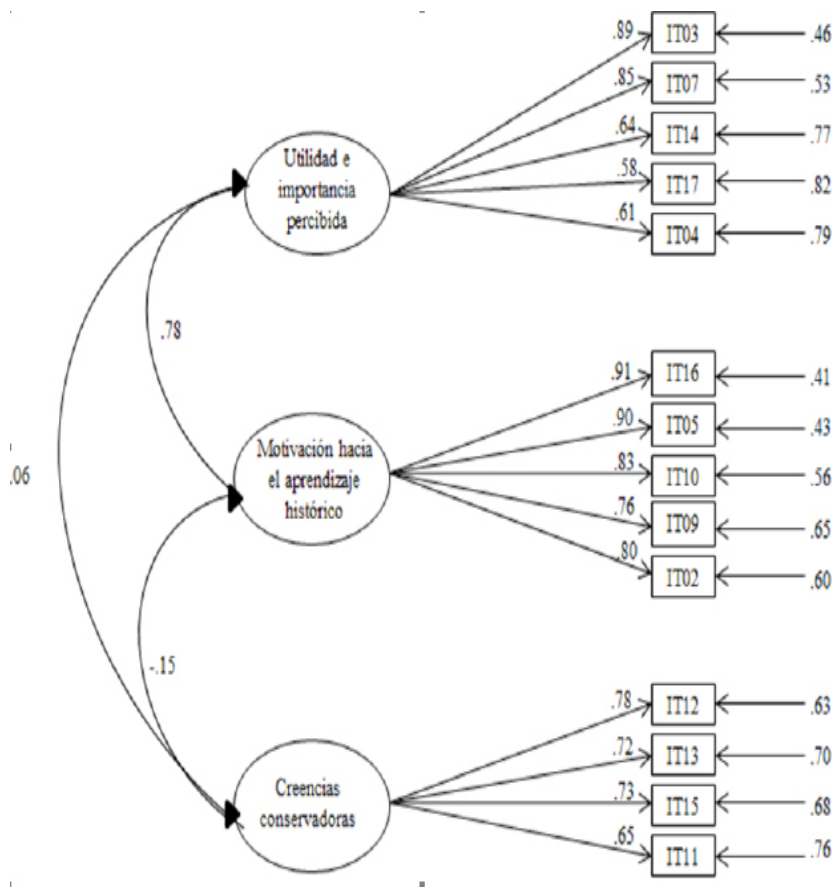

Tabla 5

Coeficientes de fiabilidad compuesta (CR) y varianza media extraída (AVE).

\begin{tabular}{llllll}
\hline Factor & $\mathbf{1}$ & $\mathbf{2}$ & $\mathbf{3}$ & $\mathbf{C R}$ & $\mathbf{A V E}$ \\
\hline $\begin{array}{l}\text { 1. Utilidad e importancia } \\
\text { percibida }\end{array}$ & $(.84)$ & .78 & -.02 & .84 & .70 \\
$\begin{array}{l}\text { 2. Motivación hacia el } \\
\text { aprendizaje histórico }\end{array}$ & & $(.93)$ & .19 & .93 & .86 \\
$\begin{array}{l}\text { 3. Creencias } \\
\text { conservadoras }\end{array}$ & & & $(.81)$ & .81 & .66 \\
\hline
\end{tabular}

\section{Discusión}

La enseñanza de la Historia ha ocupado un lugar de gran importancia en la construcción de las identidades nacionales de un buen número de Estados a lo largo de los siglos XIX, XX y XXI (López-Rodríguez, 2015). El auge del nacionalismo y de la creación de nuevos Estadosnación se encuentra íntimamente relacionado con el surgimiento y desarrollo de la disciplina de la Historia tal y como se conoce hoy en día, y su utilización con fines políticos ha sido un fenómeno global (Anderson, 1983; Barton \& Levstik, 2004; Hobsbawn, 1997; Renan, 1882) que redundaba en una politización de los programas educativos, así como de las investigaciones desarrolladas en el ámbito de la disciplina histórica y de la didáctica de la Historia (Foster, 2012; López-Rodríguez, 2015). La literatura alerta que, al contrario de la mayoría de disciplinas, no ha sido analizado el método por el cual los estudiantes se acercan a ella (Barton, 2008). Las investigaciones llevadas a cabo han versado específicamente sobre la cuestión identitaria, en áreas de conflicto social, político y/o lingüístico. Muchos son los autores que exhortan a estudiar las actitudes del alumnado para conocer la percepción de los estudiantes sobre la disciplina y sobre su propio conocimiento histórico, así como los factores que explican la génesis y evolución de dichas actitudes (Barton, 2010; Carretero, Castorina, Sarti, Van Alphen, \& Barreiro, 2013; LeTourneau, 2004;).

Sin embargo, la revisión de los instrumentos existentes para la medición de actitudes hacia la Historia nos ha llevado a encontrar un único instrumento diseñado y validado por Andrews et al. (2009, 2010), que define el constructo como constituido por dos factores independientes, que son el factor conservador y el factor liberal o multicultural, ambos bajo una misma dimensión, que es la cognitiva. No obstante, un análisis más detallado de los estudios nos permite arribar a tres conclusiones: i) la conceptualización teórica del constructo se realiza en base a un modelo unidimensional, que considera únicamente un componente cognitivo, el cual hasta el momento 
ha resultado insuficiente para explicar de manera satisfactoria las actitudes de un individuo hacia el objeto actitudinal; ii) los estudios de validación únicamente se han realizado en muestras procedentes de un contexto anglosajón, y iii) las evidencias psicométricas del mismo se han obtenido a partir de técnicas exploratorias, con lo cual no se tiene constancia de ningún estudio confirmatorio de la estructura propuesta por sus autores.

Así, tomando como punto de partida el modelo de dos componentes propuesto originalmente por Bagozzi y Burnkrant (1979), ampliamente reconocido en el ámbito del estudio de las actitudes (p. ej., Huskinson y Haddock, 2006), se planteó como objetivo de la presente investigación desarrollar y validar, mediante técnicas confirmatorias, una nueva escala para la evaluación de actitudes hacia la historia en un contexto de habla hispana.

Para ello, además del trabajo de Andrews et al. (2009, 2010), se consideró la inclusión de las dimensiones utilidad e importancia percibida e interés hacia el aprendizaje histórico, a partir de la adaptación de la escala de motivación académica (Aydin et al., 2014). De este modo, el constructo actitudes hacia la historia corresponde a un modelo de dos componentes: el afectivo, que engloba el interés hacia el aprendizaje histórico; y el cognitivo, que engloba la utilidad e importancia percibida y las creencias conservadoras del individuo.

Así, teniendo en cuenta esta conceptualización para el constructo, se propuso la confirmación de una estructura de tres factores de primer orden, agrupados bajo dos componentes de orden superior. La muestra estuvo compuesta por un total de 430 estudiantes de Enseñanza Secundaria que cursaban la asignatura de Historia. Se calcularon los índices de bondad de ajuste para el modelo teórico y se implementó una serie de mejoras sobre el mismo en base al análisis de las cargas factoriales estandarizadas, los índices de covarianza de modificación y los coeficientes de covarianza residual estandarizados. Como consecuencia, se eliminaron un total de 3 ítems de los propuestos inicialmente, con lo cual se obtuvo una versión definitiva compuesta por 14 afirmaciones, para la que el ajuste de modelo resultó bueno $\left(\mathrm{S}-\mathrm{B} \chi_{(74, \mathrm{~N}=430)}^{2}=215.54, p<.001\right.$; RMSEA [90\% IC] = .068 [.058-.079]; NNFI = .94; $\mathrm{CFI}=.95)$. Este resultado confirma la adecuación de la estructura factorial del constructo actitudes hacia la historia desde el marco conceptual del modelo de dos componentes.

Por su parte, el coeficiente alfa de Cronbach resultó ser $\alpha=.77$ para el conjunto de la escala, lo que fue indicativo de una adecuada consistencia interna de la misma (Nunnally, 1978). Asimismo, los valores de confiabilidad compuesta de cada factor resultaron superiores a los coeficientes de correlación de dicho factor con el resto, lo que permitió asegurar la validez discriminante (Bagozzi \& Yi, 2012; Henseler et al., 2015). Finalmente, los resultados de los análisis de varianza media explicada estuvieron comprendidos entre $\mathrm{AVE}=.66$ y $\mathrm{AVE}=.86$, datos que verifican la validez convergente de la nueva escala (Fornell \& Larcker, 1981).

A pesar de estos resultados prometedores, hay una serie de limitaciones metodológicas que conviene considerar en la presente investigación como punto de partida para futuras líneas de estudio a este respecto. En primer lugar, la recogida de datos fue realizada únicamente en la provincia de Bizkaia (Comunidad Autónoma Vasca, España), por lo que estos datos no pueden generalizarse directamente al resto del territorio español. No obstante, el tamaño muestral fue lo suficientemente grande y representativo de la realidad escolar vasca para poder cubrir el objetivo de la presente investigación. A este respecto, por 
tanto, se plantea como futura línea de estudio un análisis posterior de la invarianza de la estructura factorial evaluada con muestras mayores de diferentes contextos sociodemográficos. En segundo lugar, los análisis efectuados aseguran propiedades psicométricas robustas para el instrumento desarrollado, en base a evidencias de validez de constructo, consistencia interna, validez discriminante $\mathrm{y}$ validez convergente. Sin embargo, no se han incluido en el presente estudio análisis de confiabilidad test-retest que permitan asegurar la estabilidad estructural de la nueva escala, aspecto que se plantea igualmente como futura línea de trabajo.

Las evidencias de confiabilidad y validez recopiladas a lo largo del estudio permiten concluir que la escala definitiva de 14 ítems es un instrumento válido y psicométricamente robusto para la medición de las actitudes hacia la historia en estudiantes de Enseñanza Secundaria. Cabe destacar que los resultados confirman la conceptualización teórica planteada de acuerdo a la cual el constructo actitudes responde a una estructura de dos componentes de orden superior que engloban tres factores de primer orden, que son utilidad e importancia percibida, interés hacia el aprendizaje histórico y creencias conservadoras. Dicho resultado se considera una gran contribución al ámbito de investigación mencionado ya que la escala obtenida permite evaluar el componente afectivo de las actitudes hacia la historia mientras que, hasta el momento, el instrumento existente elaborado por Andrews et al. (2009, 2010) únicamente consideraba el componente cognitivo.

Asimismo, la nueva escala desarrollada, aun cuando incluye mayor cantidad de ítems que los estudios originales tomados como punto de partida, sigue siendo fácil de administrar y no supone un tiempo de cumplimentación superior a los 15 minutos. De este modo, tanto los investigadores como los docentes interesados pueden aplicarla en Enseñanza Secundaria para analizar y monitorizar las actitudes de los estudiantes hacia la historia y poder implementar adaptaciones al proceso de Enseñanza-Aprendizaje de la materia que permitan mejorar los niveles de interés o utilidad percibida, y favorecer el pensamiento liberal o multicultural. En efecto, este punto es de especial importancia dado que los estudiantes suelen pasar por alto la diversidad existente en un mismo periodo histórico entre grupos procedentes de diferentes regiones geográficas o estratos económicos, portadores de diferentes experiencias y puntos de vista. Así, las actividades curriculares planificadas por los docentes y los materiales didácticos utilizados en la asignatura de Historia pueden subrayar la diversidad $\mathrm{y}$ ayudar a los estudiantes a captar y analizar dicha diversidad entre grupos de personas del pasado (Barton, 2010).

\section{Referencias}

Ajzen, I. (1989). Attitude structure and behavior. En A. R. Pratkanis, S. J. Beckler \& A. G. Greenwald (Eds.), Attitude structure and function (pp. 241-274). Hillsdale, NJ: Lawrence Erlbaum.

Anderson, B. (1983). Imagined communities: Reflections on the origin and spread of nationalism. London: Verso.

Andrews, R., McGlynn, C., \& Mycock, A. (2009). Students' attitudes towards history: Does self-identity matter? Educational Research, 51(3), 365-377. doi: 10.1080/00131880903156948

Andrews, R., McGlynn, C., \& Mycock, A. (2010). National pride and students' attitudes towards history: An exploratory study. Educational Studies, 36(3), 299309. doi: 10.1080/03055690903424782

Auzmendi, E. (1992). Las actitudes hacia la matemáticaestadística en las enseñanzas media y universitaria: 
Características y medición. Bilbao: Mensajero.

Aydin, S., Yerdelen, S., Gürbüzoğlu-Yalmanci, S. G., \& Göksu, V. (2014). Academic Motivation Scale for Learning Biology: A scale development study. Education and Science, 39(176), 425-435. doi: https://doi.org/10.15390/eb.2014.3678

Bagozzi, R. P., \& Burnkrant, R. E. (1979). Attitude measurement and behavior change: A reconsideration of attitude organization and its relationship to behavior. En W. L. Wilkie (Ed.), Advances in Consumer Research, Vol. 6 (pp. 295-302). Ann Arbor, MI: Association for Consumer Research.

Bagozzi, R. P., Tybout, A. M., Craig, C. S., \& Sternthal, B. (1979). The construct validity of the tripartite classification of attitudes. Journal of Marketing Research, 16, 88-95. doi: 10.2307/3150879

Bagozzi, R. P., \& Yi, Y. (2012). Specification, evaluation, and interpretation of structural equation models. Journal of the Academy of Marketing Science, 40(1), 8-34. doi: 10.1007/s11747-011-0278-x

Barton, K. (2010). Investigación sobre las ideas de los estudiantes acerca de la Historia. Enseñanza de las Ciencias Sociales, 2010(9), 97-114. Recuperado de https://dialnet.unirioja.es/ejemplar/251331

Barton, K. C., \& Levstik, L. (2004). Teaching History for the Common Good. Mahwah, NJ: Lawrence Erlbaum Associates, Inc.

Barton, K. (2008). Narrative simplifications in elementary students' historical thinking. En L. Levstik \& K. Barton (Eds.), Researching history education: Theory, method, and context (pp. 181-208). New York, NY: Routledge.

Bautista, V. E., \& Mateos, D. (2011). Diseño y validación de un cuestionario de evaluación de la actitud hacia la música clásica del alumnado de educación secundaria obligatoria. Revista Electrónica de Investigación y Docencia (REID), 7, 141-161.Recuperado de http:// www.ujaen.es/revista/reid/revista/n7/REID7art8.pdf

Bentler, P. M. (1990). Comparative fit indexes in structural models. Psychological Bulletin, 107(2), 238-246. doi: 10.1037/0033-2909.107.2.238
Bentler, P. M. (2005). EQS Structural equations program manual. Encino, CA: Multivariate Software, Inc.

Bettman, J., Capon, N., \& Lutz, R. (1975). Multiattribute measurement models and multiattribute attitude theory: A test of construct validity. Journal of Consumer Research, 1(4), 1-15. doi: 10.1086/208602

Bollen, K. A. (1989). Structural equations with latent variables. New York, NY: John Wiley \& Sons.

Breckler, S. J. (1984). Empirical validation of affect, behavior, and cognition as distinct components of attitude. Journal of Personality and Social Psychology, 47(6), 1191-1205. doi: 10.1037/00223514.47.6.1191

Brown, C. H. (1983). Asymptotic comparison of missing data procedures for estimating factor loadings. Psychometrika, 48(2), 269-292. doi: 10.1007/ bf02294022

Brown, T. (2006). Confirmatory factor analysis for applied research. New York: Guildford.

Browne, M. W., MacCallum, R. C., Kim, C. T., Andersen, B. L., \& Glaser, R. (2002). When fit indices and residuals are incompatible. Psychological Methods, 7(4), 403-421. doi: 10.1037//1082-989X.7.4.403

Burke, K. (1984). Attitudes toward history. California: University of California Press.

Carretero, M., Castorina, J. A., Sarti, M., Van Alphen, F., \& Barreiro,A. (2013). La Construcción del conocimiento histórico. Propuesta Educativa, 22(39), 13-23. Recuperado de http://www.propuestaeducativa. flacso.org.ar/archivos/articulos/35.pdf

Casal, S. (2011). Aprender historia en la escuela secundaria: El caso de Morelia, Michoacán (México). Revista Mexicana de Investigación Educativa, 16(48), 73105.

Chou, C. P., \& Bentler, P. M. (1995). Estimates and tests in structural equation modeling. En R. H. Hoyle (Ed.), Structural equation modeling: Concepts, issues, and applications. Thousand Oaks, CA: Sage Publications, Inc.

Clark, L. A., \& Watson, D. (1995). Construct validity: Basic issues in objective scale development. Psychological 
Assessment, 7(3), 309-319. doi: 10.1037/10403590.7.3.309

DeVellis, R. F. (2012). Scale development: Theory and applications $\left(3^{\mathrm{a}}\right.$ ed.). Thousands Oaks, CA: Sage Publications, Inc.

Esses, V. M., Haddock, G., \& Zanna, M. P. (1993). Assessing the structure of prejudicial attitudes: The case of attitudes toward homosexuals. Journal of Personality and Social Psychology, 65(6), 11051118. doi: 10.1037/0022-3514.65.6.1105_

Fennema, E., \& Sherman, J. A. (1976). FennemaSherman Mathematics Attitudes Scales: Instruments designed to measure attitudes toward the learning of Mathematics by females and males. Journal of Research in Mathematics Education, 7(5), 324-326. doi: $10.2307 / 748467$

Finney, S. J., \& DiStefano, C. (2006). Non-normal and categorical data in SEM. En G. R. Hancock \& R. O. Mueller (Eds.). Structural equation modeling: A second course (pp. 269-314). Greenwich, CT: Information Age Publishing.

Fishbein, M. (1963). An investigation of the relationship between beliefs about an object and the attitude toward that object. Human Relations, 16(3), 233240. doi: $10.1177 / 001872676301600302$

Fishbein, M. (1967). A behavior theory approach to the relations between beliefs about an object and the attitude toward the object. En M. Fishbein (Ed.), Readings in attitude theory and measurement (pp. 389-400). New York: Wile.

Fishbein, M., \& Ajzen, I. (1974). Attitudes toward objects as predictors of single and multiple behavioral criteria. Psychological Review, 81(1), 59-74. doi: 10.1037/ h0035872

Floyd, F. J., \& Widaman, K. (1995). Factor analysis in the development and refinement of clinical assessment instruments. Psychological Assessment, 7(3), 286299. doi: 10.1037/1040-3590.7.3.286

Fornell, C., \& Larcker, D. F. (1981). Evaluating structural equation models with unobservable variables and measurement error. Journal of Marketing Research,
18(1), 39-50. doi: 10.2307/3151312

Foster, S. (2012). Re-thinking historical textbooks in a globalised world. En M. Carretero, M. Asensio \& M. Rodríguez-Moneo (Eds.), History Education and the Construction of National Identities (pp. 49-62). Charlotte CT: Information Age Publishing.

Fuentes-Moreno, C. (2002). La visión de la historia por los adolescentes: Revisión del estado de la cuestión en Estados Unidos y el Reino Unido. Enseñanza de las Ciencias Sociales, 2002(1), 55-68. Recuperado de https://dialnet.unirioja.es/ejemplar/70981

Gairín, J. (1990). Las actitudes en educación. Un estudio sobre la educación matemática. Barcelona: Boixareu Universitaria.

Hair, J. F., Black, W. C., Babin, B. J., \& Anderson, R. E. (2010). Multivariate data analysis ( $7^{\mathrm{a}}$ ed.). Upper Saddle River, NJ: Prentice Hall.

Hawkins, D. I., Mothersbaugh, D. L., \& Best, R. J. (2007). Consumer behavior: Building marketing strategy. Boston: McGraw-Hill/Irwin.

Henseler, J., Ringle, C. M., \& Sarstedt, M. (2015). A new criterion for assessing discriminant validity in variance-based structural equation modeling. Journal of Academy of Marketing Science, 43(1), 115-135. doi: 10.1007/s11747-014-0403-8

Hobsbawm, E. (1997). Nations and Nationalism since 1780: Programme, myth, reality. Cambridge: Cambridge University Press.

Hoyle, R. H., \& Panter, A. T. (1995). Writing about structural equation models in structural equation modeling. En R. H. Hoyle (Ed.), Structural Equation Modeling: Concepts. Issues and Applications (pp. 158-176). Thousand Oaks, CA: Sage Publications, Inc.

Hu, L. T., \& Bentler, P. M. (1999). Cutoff criteria for fit indices in covariance structure analysis: Conventional criteria versus new alternatives. Structural Quotation Modeling, 6(1), 1-55. doi: 10.1080/10705519909540118

Huguet, A. (2005). Génesis y desarrollo de las actitudes lingüísticas en contextos bilingües. Análisis de algunas variables del ámbito escolar y sociofamiliar. 
Revista de Psicología Social, 20(2), 175-191. doi: 10.1174/0213474053683167

Huskinson, T. L. H., \& Haddock, G. (2006). Individual differences in attitude structure and the accessibility of the affective and cognitive components of attitude. Social Cognition, 24(4), 453-468. doi: 10.1521/ soco.2006.24.4.453

IBM Corp. (2013). IBM SPSS Statistics for Windows, Version 22.0. [software de cómputo]. Armonk, NY: IBM Corp.

Kline, R. B. (2005). Principles and practice of structural equation modeling ( $2^{\mathrm{a}}$ ed.). New York, NY: The Guilford Press.

Kölbl, C., \& Straub, J. (2001). Historical consciousness in youth: Theoretical and exemplary empirical analysis. Forum: Qualitative Social Research, 2(3).

Kothandapani, V. (1971). Validation of feeling, belief, and intention to act as three components of attitude and their contribution to prediction of contraceptive behavior. Journal of Personality and Social Psychology, 19, 321-333. doi: 10.1037/h0031448

Kraus, S. J. (1995). Attitudes and prediction of behaviour: A meta-analysis of the empirical literature. Personality and Social Psychology Bulletin, 21(1), 58-75. doi: 10.1177/0146167295211007

Lasagabaster, D. (2003). Trilingüismo en la enseñanza. Actitudes hacia la lengua minoritaria, la mayoritaria y la extranjera. Lleida: Milenio.

Lei, M., \& Lomax, R. G. (2005). The effect of varying degrees of nonnormality in structural equation modeling. Structural Equation Modeling: A Multidisciplinary Journal, 12, 1-27. doi: 10.1207/ s15328007sem1201_1

LéTourneau, J., \& Moisan, S. (2004). Young people's assimilation of a collective historical memory: A case study of Quebeckers of French-Canadian heritage. En P. Seixas (Ed.), Theorizing historical consciousness, (pp. 109-128). Toronto: University of Toronto Press.

Ley Orgánica 15/1999, de 13 de diciembre, de Protección de Datos de Carácter Personal. Boletín Oficial del
Estado, núm. 298, de 14 de diciembre de 1999, pp. 43088-43099. Recuperado de https://www.boe.es/ boe/dias/1999/12/14/pdfs/A43088-43099.pdf

Lim, S. Y., \& Chapman, E. (2013). Development of a short form of the Attitudes Toward Mathematics Inventory. Educational Studies in Mathematics, 83(1), 145-164. doi: 10.1007/s10649-012-9414-X

López-Rodríguez, C. (2015). Aprendizaje de la historia e identidades nacionales: El punto de vista de los estudiantes. CLIO. History and History teaching, 41. Recuperado de http://clio.rediris.es

Marin, G., \& Marin, B. V. (1991). Research with Hispanic populations. Newbury Park, CA: Sage Publications, Inc.

Marsh, H. W., Hau, K. T., \& Wen, Z. (2004). In search of golden rules: Comment on hypothesis-testing approaches to setting cutoff values for fit indexes and dangers in overgeneralizing $\mathrm{Hu}$ and Bentler's findings. Structural Equation Modeling, 11, 320341. doi: 10.1207/s15328007sem1103_2

Mazis, M. B., Ahtola, O. T., \& Klippel, R. E. (1975). A comparison of four multi-attribute models in the prediction of consumer attitudes. Journal of Consumer Research, 2(1), 38-52. doi: 10.1086/208614

McGuire, W. J. (1989). Theoretical foundations of campaigns. En R. Rice \& C. Atkin (Eds.), Public communication campaigns (pp. 43-65). Newberry Park, CA: Sage.

Millar, M. G., \& Millar, K. U. (1990). Attitude change as a function of attitude type and argument type. Journal of Personality and Social Psychology, 59, 217-228. doi: 10.1037/0022-3514.59.2.217

Multivariate Software (2012). EQS 6.1 [software de cómputo]. Disponible en http://www.mvsoft.com/ index.htm

Muñiz, J., Elosua, P., \& Hambleton, R. K. (2013). Directrices para la traducción y adaptación de los tests: Segunda edición. Psicothema, 25, 149155. Recuperado de http://www.psicothema.com/ psicothema.asp? $\mathrm{id}=4093$

Nunnally, J. C. (1978). Psychometric theory (2 $2^{\mathrm{a}}$ ed.). New 
York, NY: McGraw-Hill.

Ostrom, T. M. (1969). The relationship between the affective, behavioral, and cognitive components of attitude. Journal of Experimental Social Psychology, 5, 12-30. doi: 10.1016/0022-1031(69)90003-1

Pietsch, J., Walker, R., \& Chapman, E. (2003). The relationship among self-concept, self-efficacy, and performance in Mathematics during secondary school. Journal of Educational Psychology, 95(3), 589-603. doi: 10.1037/0022-0663.95.3.589

Pett, M. A., Lackey, N. R., \& Sullivan, J. J. (2003). Making sense of factor analysis: The use of factor analysis for instrument development in health care research. Thousand Oaks: Sage Publications, Inc.

Renan, E. (1882). What is a Nation? En H. K. Bhabha (1990) (Ed.), Nation and Narration (pp. 8-22). Routledge: London.

Rosenberg, M. J., \& Hovland, C. I. (1960). Cognitive, affective and behavioral components of attitudes. En M. J. Rosenberg, C. I. Hovland, W. J. McGuire, R. P. Abelson \& J. W. Brehm (Eds.), Attitude Organization and Change: An Analysis of Consistency Among Attitude Components. New Haven: Yale University Press.

Sandman, R. S. (1980). The Mathematics Attitude Inventory: Instrument and user's manual. Journal of Research in Mathematics Education, 11(2), 148-149. doi: $10.2307 / 748906$

Satorra, A. (2003). Power of chi-square goodness-of-fit test in structural equation models: The case of nonnormal data. En H. Yanai, A. Okada, K. Shigemasu, Y. Kano \& J. J. Meulman (Eds.), New developments of psychometrics (pp. 57-68). Tokio: Springer Verlag.

Satorra, A., \& Bentler, P. M. (2001). A scaled difference chisquare test statistic for moment structure analysis. Psychometrika, 66(4), 507-514. doi: 10.1007/ BF02296192

Sheth, J. N. (1974). A field study of attitude structure and attitude-behavior relationship. En J. N. Sheth (Ed.), Models of Buyer Behavior (pp. 242-268). New York: Harper and Row.
Tabachnick, B. G., \& Fidell, L. S. (2007). Using multivariate statistics ( $5^{\mathrm{a}} \mathrm{ed}$.). Boston, MA: Allyn \& Bacon.

Tapia, M., \& Marsh, G. E. (2004). An instrument to measure mathematics attitudes. Academic Exchange Quarterly, 8(2), 16-21.

Tarraga, R. (2008). Relación entre rendimiento en solución de problemas $y$ factores afectivos motivacionales en alumnos con y sin dificultades del aprendizaje. Apuntes de Psicología, 26, 143-148. Recuperado de https://dialnet.unirioja.es/servlet/ articulo? codigo $=2674459$

Teitsma, J. (2010). The effects of popular and classical music aural discrimination training on the aural discrimination skills of middle school students. New Jersey: University of New Jersey.

Thurstone, L. L. (1928). Attitudes can be measured. American Journal of Sociology, 33(4), 529-554. doi: $10.1086 / 214483$

Tuncalp, S., \& Sheth, J. N. (1975). Prediction of attitudes: A comparative study of Rosenberg, Fishbein, and Sheth models. En M. J. Schlinger (Ed.), Advances in Consumer Research, Vol. 2. Chicago: Association for Consumer Research.

Ullman, J. B. (2006). Structural equation modeling. En B. G. Tabachnick \& L. S. Fidell (Eds.), Using multivariate statistics ( $5^{\mathrm{a}}$ ed.) (pp. 653-771). Boston, MA: Allyn \& Bacon.

Verplanken, B., Hofstee, G., \& Janssen, H. J. W. (1998). Accessibility of affective versus cognitive components of attitudes. European Journal of Social Psychology, 28, 23-35. doi: 10.1002/(sici)10990992(199801/02)28:1<23::aid-ejsp843>3.3.co;2-q

Von Borries, B. (1994). (Re-)constructing history and moral judgment: On relationships between interpretations of the past and perceptions of the present. En F. Voss \& M. Carretero (Eds.), Cognitive and instructional processes in History and the Social Sciences, (pp. 339-355). Hillsdale, NJ: Erlbaum.

Wertsch, J. V. (1994). Struggling with the past: Some dynamics of historical representation. En J. P. Voss \& M. Carretero (Eds.), Cognitive and instructional 
processes in History and the social sciences, (pp. doi: 10.1007/s10649-006-9028-2

223-238). Hillsdale, NJ: Erlbaum.

Wilkie, W. L., \& Pessemier, E. A. (1973). Issues in marketing's use of multi-attribute models. Journal of Marketing Research, 10, 428-441. doi: $10.2307 / 3149391$

Woodmansee, J. J., \& Cook, S. W. (1967). Dimensions of verbal racial attitudes: Their identification and measurement. Journal of Personality and Social Psychology, 7, 240-250. doi: 10.1037/h0025078

Zan, R., Brown, L., Evans, J., \& Hannula, M. (2006). Affect in Mathematics Education: An Introduction. Educational Studies in Mathematics, 63, 113-121.

\begin{tabular}{|c|c|}
\hline Ítem & Afirmación \\
\hline 01 & $\begin{array}{l}\text { Las minorías étnicas deberían ser apoyadas para que preserven su historia y su cultura, y ello debería quedar } \\
\text { reflejado en las clases de Historia }\end{array}$ \\
\hline 02 & Disfruto debatiendo sobre temas históricos \\
\hline 03 & No veo razón por la que aprender Historia \\
\hline 04 & Considero que las cosas que aprendo en Historia son útiles \\
\hline 05 & Disfruto aprendiendo temas históricos \\
\hline 06 & La inmigración y la globalización han reforzado mi comprensión del lugar donde vivo \\
\hline 07 & No sé por qué debo estudiar Historia \\
\hline 08 & $\begin{array}{l}\text { Los inmigrantes que buscan la nacionalidad deberían pasar una prueba sobre nuestra historia cultural y } \\
\text { nacional }\end{array}$ \\
\hline 09 & Disfruto aprendiendo sobre los temas históricos en los que estoy interesado/a \\
\hline 10 & Disfruto compartiendo los nuevos aspectos históricos que aprendo \\
\hline 11 & Los profesores de Historia deben mostrar lealtad al Estado y promover una visión positiva de nuestro país \\
\hline 12 & $\begin{array}{l}\text { Algunas partes de la Historia de mi país no deberían ser enseñadas ya que son ofensivas hacia algunos } \\
\text { ciudadanos por motivos religiosos o políticos }\end{array}$ \\
\hline 13 & $\begin{array}{l}\text { La religión debería ser la dimensión más importante a la hora de enseñar la Historia de nuestro país, y } \\
\text { tendría que estar siempre presente }\end{array}$ \\
\hline 14 & No creo que los temas que estudiamos en Historia sean importantes para mi futuro \\
\hline 15 & $\begin{array}{l}\text { El Estado debería tener el control sobre lo que se enseña en las clases de Historia para asegurar que todos los } \\
\text { ciudadanos tendrán la misma visión de la identidad nacional y el mismo sentido de lealtad hacia el país }\end{array}$ \\
\hline 16 & Me interesan los temas históricos \\
\hline 17 & No me gusta participar en las actividades de la clase de Historia \\
\hline
\end{tabular}

